\title{
Optical absorption coefficients of gold nanorods through acoustic waves in an optical hyperthermia system
}

\author{
P.F. Escudero ，C. Sánchez ，F. del Pozo ， J.J. Serrano
}

\begin{abstract}
The irradiation of small suspensions of gold nanorods (GNRs) using laser pulses at $808 \mathrm{~nm}$ and $25 \mathrm{KHz}$, produces a series of effects occurring during the interaction of each laser pulse with the amount of gold nanorods in suspension. The result of the interaction laser-GNRs is the generation of acoustic waves, which may influence on cell death in an optical hyperthermia therapy system. Therefore the identification and characterization of acoustic signals and their absorption coefficients is performed in a phantom based in a neutral gelatin system, in order to able to identify and quantify the influence of these acoustic waves in cell death in optical hyperthermia essays.
\end{abstract}

Keywords- Optical hyperthermia, gold nanorods, optoacoustic spectroscopy, pressure waves, absorption coefficient.

\section{INTRODUCTION}

The optical absorption property of gold nanorods has been researched extensively for biomedical applications [I] [2].

This absorption is produced by several physical effects as surface plasmon resonance, synchronization wavelengthnanorods dimensions while these are in suspension being interacting with a laser beam [3]. These physical effects are characterized by the sum of many effects produced by each individual nanorod [4] [5].

The surface plasmon is a phenomenon characterized by the presence of free electrons on the surface of the each gold nanorod, by the interaction with photons; these free electrons produce the surface plasmon resonance. This resonance is considered the principal contributor to the optical absorption, and it is also considered in the contribution for the acoustic wave generation. Since absorption is higher in the near infrared band (NIR), this can be used to improve the penetration of light into tissue [3][5] [6].

According these features, gold nanorods have been used in optical hyperthermia essays. These are used to define and localize treatment areas where thermal gradients are generated between $41^{\circ} \mathrm{C}$ and $43^{\circ} \mathrm{C}$ during the time considered necessary to induce irreversible damage [7] [8].

Thermal gradients have been obtained through dimensions of gold nanorods, length and diameter with the wave length laser synchronization. This synchronization was used to dispose of a noninvasive technic, obtaining the maximum optical absorption of the nanorods. [9] [10] [11].

Our research group has initially experimented to produce optical hyperthermia using a laser of continuous light, however several techniques have been developed with pulsed lasers in the femtosecond pulse range, which are used to generate optical hyperthermia with a better precision to localize the treatment area [9].

In this work, we study the identification and characterization of the optical absorption coefficient $\mu_{a b s}$ produced by a small volume of gold nanorods in suspension. The $\boldsymbol{\mu}_{a b s}$ are calculated from the acoustic waves captured from a phantom based system by the interaction of a small volume of nanorods and modulated pulses of a laser.

By this characterization, we expected to contribute to identify each effect feature that produces the cell death in optical hyperthermia treatment trials of cancer tissue.

\section{MATERIALS AND METHODS}

The basis of the optical hyperthermia system is constituted of a continuous wave laser at $808 \mathrm{~nm}$ wavelength $(5 \mathrm{~W}$ MDL-H-808, H-PSU-LED power source; Changchun New Industries), and gold nanorods (0-10-808 Nanorodz; Nanopartz, Salt Lake City, UT), $10 \mathrm{~nm}$ axial diameter and 41nm length.

We have built a modification of this system to capture sound waves based in a phantom of neutral gelatin according to the concentration of GNRs and output power of a laser modulated at $25 \mathrm{KHz}$.

The system Fig. 1 allows the detection of acoustic waves from a small volume of GNRs embedded in a neutral gelatin phantom coupled directly to a piezoelectric ultrasonic sensor. 


\section{A. Phantom based system for detection of acoustic waves.}

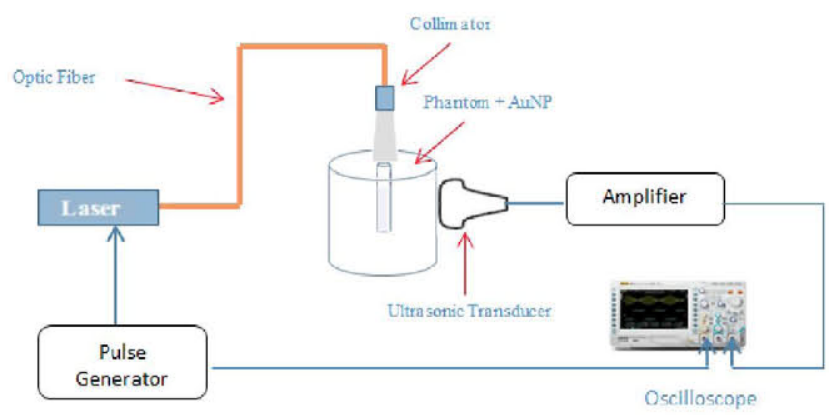

Fig. 1 Ultrasonic detection system based on a Phantom as a variation from the basic optical hyperthermia system reported in [7]

\section{Description of the System}

The system of the Fig. 2 shows the full experimental detection system of acoustic waves. This was performed from the basic hyperthermia system that was used in hyperthermia researches to produce glioblastoma cell death [7].

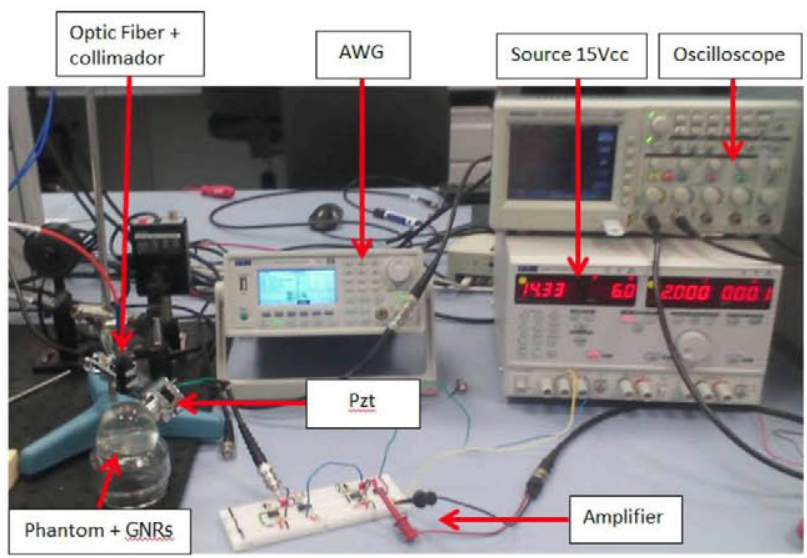

Fig. 3 Full experimental detection system of acoustic waves.

The ultrasonic detection system based on a Phantom is described as:

Emission of laser pulses: The continuous wave laser is modulated at $25 \mathrm{KHz}$, using an arbitrary waveform generator (AWR, TG5011) in a range of $30 \%$ to $70 \%$ duty cycles, considering three levels output power for each duty cycle, 250,550 and $850 \mathrm{~mW}$.

Gold Nanorods: $10 \mathrm{~nm}$ axial diameter and $41 \mathrm{~nm}$ length GNRs are used.

Phantom: Used as a repository of GNRs. It is built with an internal cavity of $2 \times 15 \mathrm{~mm}$ diameter and height respectively. External dimensions are $52 \times 50 \times 22 \mathrm{~mm}$ base diame- ter, height and cover respectively. It is made of neutral gelatin with optical absorption negligible at $808 \mathrm{~nm}$ wavelength. The cross-section and penetration deep is $15 \mathrm{~mm}$ approximately, irradiating from the top.

Reception: The acoustic waves are captured by immersion sensor (Kobitone TM), at $25 \mathrm{KHz}$ peak of resonance.

Amplification: The acoustic waves are amplified to 60 $\mathrm{dB}$. This amplifier has a sensitivity of $500 \mathrm{nV}$ of input electric potential to fit into the acoustic waves are in the range of $500 \mathrm{nV}$ to $2.5 \mu \mathrm{V}$. This signal amplified is also averaged using 128 frames for a noise reduction of the acoustic wave.

Visualization: To visualize the captured acoustic wave an oscilloscope (Tektronix TDS 2024B) was used. It allows $100 \mathrm{MS} / \mathrm{s}$ in four channels.

\section{RESULTS AND DISCUSSION}

\section{A. Identification of acoustic waves}

We used a laser beam at frequencies of $3 \mathrm{KHz}$ and $5 \mathrm{KHz}$, $20 \mu$ s pulse width, and $200 \mu$ s gap between pulses. A suspension of $0.0157 \mathrm{~mm}^{3}$ of GNRs is irradiated and the acoustic signals generated are detected by the presence of a main peak, which could represent the acoustical wave front interacting with the ultrasonic sensor surface Fig. 4.

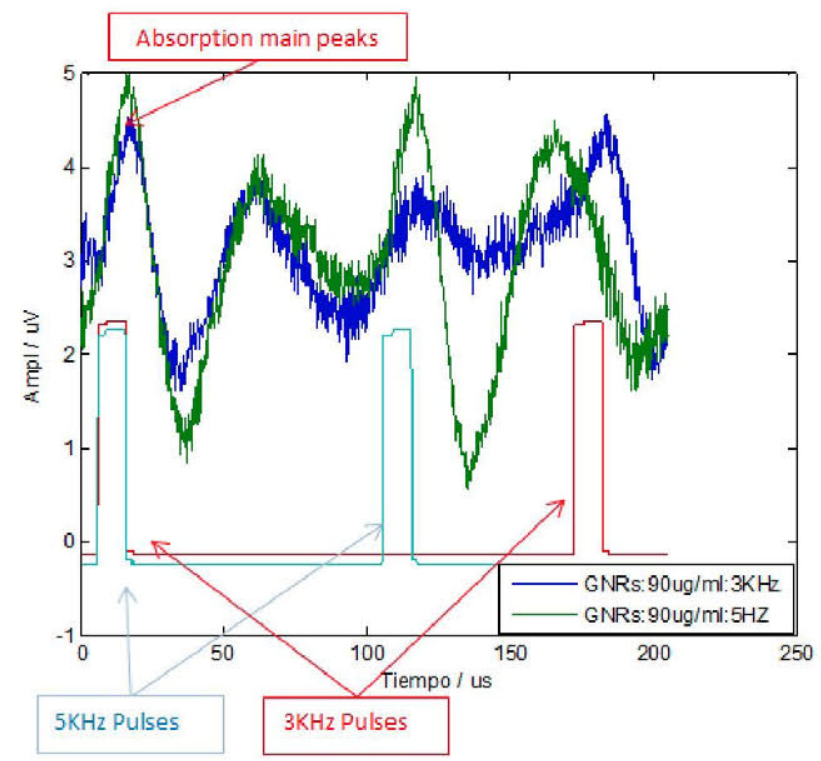

Fig. 4 Acoustic waves comparison in time. ( $3 \mathrm{KHz}$ and $5 \mathrm{KHz}$ ) frequencies used to identify the variation of the wave period versus the variation of the distance between the laser source and the phantom.

The $3 \mathrm{KHz}$ acoustic signal in blue in Fig. 4 includes the full-wave and damping, as opposed to the acoustic signal at 
$5 \mathrm{KHz}$ green. Only the first harmonic is displayed overlapping with the next harmonic.

To characterize individual acoustic waves, we performed an analysis in the time domain, which corresponded to the amount of energy absorbed by the suspension of nanorods. The Time of Flight (ToF) is compared with the laser shot pulse, and the laser pulse modulation Fig. 5. To identify the variation in time, the laser beam is shoot until the acoustic wave is generated.

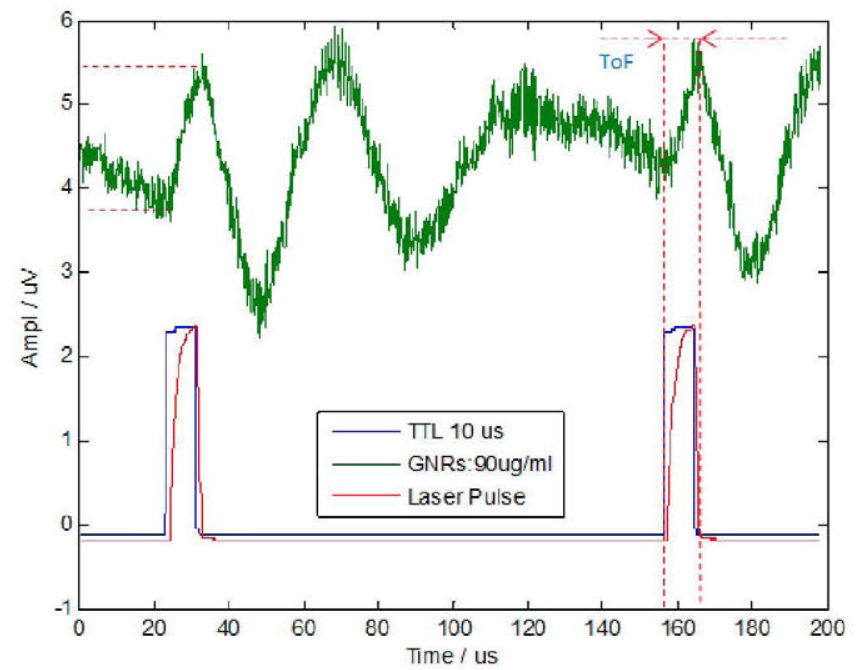

Fig. 5 Comparison: Laser pulse shot, acoustic signal, TTL.

To calculate the ToF which is the time of travel of the acoustic wave to reach the transducer surface from nanorods suspension inside the phantom, the speed of sound in water is assumed to be about $1.5 \mu \mathrm{s} / \mathrm{mm}$. Then,

$$
\begin{aligned}
& \text { ToF }=\text { dis } \tan c e * V_{\text {s-water }} \\
& \text { ToF }=30 \mathrm{us}
\end{aligned}
$$

After generating, capturing and quantifying the acoustic waves in this manner, they are generated by sum of different effects occurring in the irradiated suspension of nanorods:

- Thermal expansion by the interaction of the entire nanorods in suspension volume with each pulse of laser, shaping the acoustic wave response in the time domain.

- Contribution of the plasmon resonance effect.

\section{B. Calculation of absorption coefficients}

To quantify the absorption coefficient $\boldsymbol{\mu}_{a b s}$ of the GNRs in suspension embedded in neutral gelatin phantom we use.

$$
I=I_{0} e^{\left(-\mu_{a b s} d\right)}
$$

Where the fluency of the laser beam $I_{0}$ initial intensity, and a final intensity $\boldsymbol{I}$, transmitted light intensity and decaying exponentially with the increasing concentration of the suspension of GNRs

From this equation it follows that the energy density absorbed $I_{a b s}=I_{0}-I$. From this equation we can see that $\boldsymbol{I}_{\boldsymbol{a b s}}$ is maximum $\boldsymbol{I}_{\boldsymbol{a b s}-\max }$, when $\boldsymbol{I}=\mathbf{0}$. Then following the physical principle of the optical-acoustic effect it is deduced that $\boldsymbol{I}_{\text {abs-max }}$ induced by suspension of GNRs, the maximum pressure $\boldsymbol{P}_{\max }$ is obtained. This maximum pressure is represented by:

$$
P_{\max }=\Gamma I_{a b s-\max }=\Gamma I_{0}
$$

Where $\boldsymbol{I}_{\mathbf{0}}$ is the output power of the laser in $\left[\mathrm{Ws} / \mathrm{cm}^{2}\right]$.

Using the generated pressure in function of the GNRs absorption, it can be expressed as:

$$
\begin{gathered}
P=P_{\max }\left(1-e^{-\mu_{a b s} d}\right), \\
\text { where, } \mu_{a b s}=\frac{-1}{d} \ln \left(\frac{P}{P_{\max }}\right)
\end{gathered}
$$

Considering that the amplitude of the acoustic wave is directly proportional to the amplitude of voltage produced by the piezoelectric sensor, we have:

$$
\mu_{a b s}=\frac{-1}{d} \ln \left(\frac{V}{V_{\max }}\right)
$$

Where, $\boldsymbol{d}$ is the cross section of the volume of GNRs. Using this expression we proceed to calculate the $\mu_{a b s}$, of the GNRs from the acoustic signals obtained in the system based on a phantom, shown on Fig. 6.

In the Fig. 6. We could identify the variation of $\boldsymbol{\mu}_{a b s}$, for three concentrations $(54,72$, and $90 \mu \mathrm{g} / \mathrm{ml})$ of nanorods in suspension, and the variation for each levels of output power for each duty cycle. The analysis about the optical abortion behavior according the irradiation with several output power of laser is reported in [3]. 


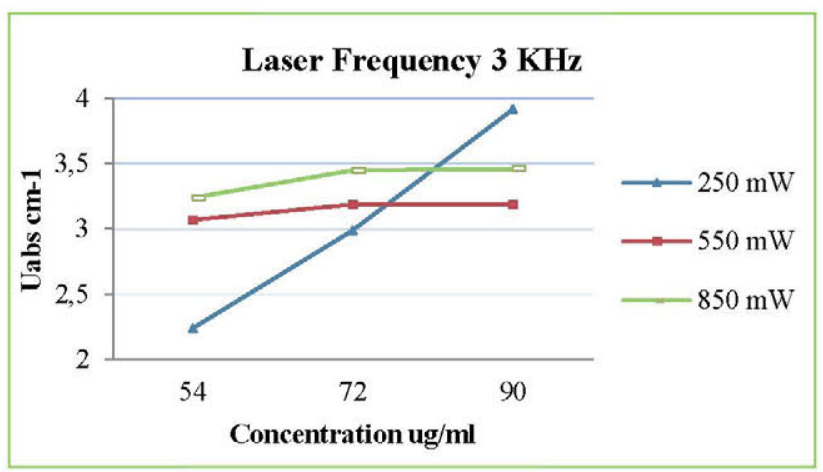

Fig. 6 Comparison of the optical absorption level in $\mathrm{cm}^{-1}$ according to the concentration of gold nanorods and output power of laser, $\mu_{a b s}$ at $3 \mathrm{KHz}$

The electrical potential detected by the ultrasonic sensor is directly proportional to the amount of light absorbed by the medium (GNRs).

In our discussion it has been considered that the scattering coefficient $\boldsymbol{\mu}_{\text {sca }}$ is negligible because the acoustic waves are generated by the amount of laser light absorbed by GNRs in suspension.

\section{CONCLUSIONS}

With the use of a phantom made of neutral gelatin we can achieve the highest possible absorption of laser radiation beam in GNRs, because the material from which the phantom is fabricated has an absorption coefficient that is negligible at $808 \mathrm{~nm}$ wavelength. This is the same laser wavelength used in the optical hyperthermia essays. This phantom feature allowed us to characterize the sound waves captured by the ultrasonic sensor, and quantify the absorption coefficient for each suspension densities of GNRs.

The results presented, present a great contribution to the process of identification and characterization of the physical effects that occur in the GNRs irradiated with a beam of laser light. It is expected that the technique could be useful in the identification of contribution of each of these effects in cell death assays in optical hyperthermia treatment.

\section{CONFLICT OF INTEREST}

"The authors declare that they have no conflict of interest".

\section{REFERENCES}

1. Horacio Lamela Vincent Cunningham, "Optical and optoacoustic measurements of the absorption properties of spherical gold nanoparticles within a highly scattering medium," Optics \& Laser Technology, vol. 42, pp. 769-774, 2010.

2. Vincent Cunningham, Daniel C. Gallego Horacio Lamela, "Experimental study using optoacoustic spectroscopy (OAS) on spherical gold nanoparticles," Optics \& Laser Technology, vol. 43, pp. 143-146, 2011.

3. J. A. Ramos, T. Fernández, F. del Pozo, J. J. Serrano C. Sánchez, "Photoacoustics of Gold Nanorods under Low Frequency Laser Pulses in Optical Hyperthermia," IFMBE Proceedings, vol. 41, pp. 868-871, 2014.

4. Ling Tong, Yan Zhao, Matthew N Hansen, Jin-Xin Cheng \& Alexander Wei Terry B Huff, "Hyperthermic effects of gold nanorods on tumor cells," Nanomedicine, vol. 2, no. 1, pp. 121$132,2007$.

5. Leonid Vigderman, Eugene R. Zubarev, and Chaoyang Jiang Ying Bao, "Robust Multilayer Thin Films Containing Cationic Thiol-Functionalized Gold Nanorods for Tunable Plasmonic Properties," Langmuir, vol. 28, pp. 923-930, 2011.

6. Xiaoshan Kou , Zhi Yang and Jianfang Wang Weihai Ni, "Tailoring Longitudinal Surface Plasmon Wavelengths, Scattering and Absorption Cross Sections of Gold Nanorods," ACS Nano, vol. 2, no. 4, pp. 677-686, 2008.

7. Cristina Sanchez Lopez de Pablo, Alberto Martinez Serrano, Francisco del Pozo Guerrero, Jose Javier Serrano Olmedo, Milagros Ramos Gomez Tamara Fernandez Cabada, "Induction of cell death in a glioblastoma line by hyperthermic therapy based on gold nanorods," International Journal of Nanomedicine, vol. 2012, no. 7, pp. 1511-1523, 2012.

8. Park S, Aglyamov S, Larson T, Ma L, Sokolov K, Johnston K, Milner T, Emelianov SY Shah J, "Photoacoustic imaging and temperature measurement for photothermal cancer therapy," $J$ Biomed Opt, vol. 13, no. 3, p. 034024, 2008.

9. Wang X, Hartland GV, Mulvaney P, Juste JP, Sader JE Hu M, "Vibrational response of nanorods to ultrafast laser induced heating: theoretical and experimental analysis," J Am Chem Soc, vol. 125 , no. 48, pp. 14925-33, 2003.

10. Julio Alberto Ramos, Tamara Fernández, Milagros Ramos, Alberto Martínez, Francisco del Pozo, José Javier Serrano Cristina Sánchez, "Photoacoustic effect measurement in aqueous suspensions of gold nanorods caused by low-frequency and lowpower near-infrared pulsing laser irradiation," Applied Optics, vol. 52, no. 19, pp. 4698 - 4705, 2013.

11. Ser-Sing Chang, Chien-Liang Lee, and C. R. Chris Wang YuYing, "Gold nanorods: electrochemical synthesis and optical properties," J. Phys. Chem. B, vol. 101, no. 34, pp. 6661-6664, 1997. 